\title{
The relationship between renal warm ischemia time and glomerular loss. An experimental study in a pig model ${ }^{1}$
}

\author{
José Aurelino Damasceno-Ferreira', Gustavo Ruschi Bechara", Waldemar Silva Costa"', Marco \\ Aurélio Pereira-Sampaio ${ }^{\mathrm{IV}}$, Francisco José Barcellos Sampaiov , Diogo Benchimol De Souzavı
}

\begin{abstract}
'Master, Postgraduate Program in Physiopathology and Surgical Sciences, Urogenital Research Unit, Universidade Estadual do Rio de Janeiro (UERJ). Assistant Professor, Department of Veterinary Clinical Pathology, Universidade Federal Fluminense (UFF), Niteroi-RJ, Brazil. Conception and design of the study, acquisition and interpretation of data, final approval.

"Master, Postgraduate Program in Physiopathology and Surgical Sciences, Urogenital Research Unit, UERJ, Rio de Janeiro-RJ, Brazil. Acquisition and interpretation of data, manuscript preparation, final approval.

I'PhD, Visiting Researcher, Postgraduate Program in Physiopathology and Surgical Sciences, Urogenital Research Unit, UERJ, Rio de Janeiro-RJ, Brazil. Conception and design of the study, acquisition and interpretation of data, final approval.

IvPhD, Associate Professor, Postgraduate Program in Physiopathology and Surgical Sciences, Urogenital Research Unit, UERJ, Rio de Janeiro-RJ. Associate Professor, Department of Morphology, UFF, Niteroi-RJ, Brazil. Conception and design of the study, acquisition and interpretation of data, manuscript preparation, final approval.

'PhD, Full Professor, Head, Postgraduate Program in Physiopathology and Surgical Sciences. Head, Urogenital Research Unit, UERJ, Rio de Janeiro-RJ, Brazil. Conception and design of the study, acquisition and interpretation of data, final approval.

VIPhD, Associate Professor, Postgraduate Program in Physiopathology and Surgical Sciences, Urogenital Research Unit, UERJ, Rio de Janeiro-RJ, Brazil. Conception and design of the study, acquisition and interpretation of data, manuscript preparation, final approval.
\end{abstract}

\begin{abstract}
Purpose: To investigate the glomerular number after different warm ischemia times.

Methods: Thirty two pigs were assigned into four groups. Three groups (G10, G20, and G30) were treated with 10, 20, and 30 minutes of left renal warm ischemia. The sham group underwent the same surgery without renal ischemia. The animals were euthanized after 3 weeks, and the kidneys were collected. Right kidneys were used as controls. The kidney weight, volume, cortical-medullar ratio, glomerular volumetric density, volume-weighted mean glomerular volume, and the total number of glomeruli per kidney were obtained. Serum creatinine levels were assessed pre and postoperatively.

Results: Serum creatinine levels did not differ among the groups. All parameters were similar for the sham, G10, and G20 groups upon comparison of the right and left organs. The G30 group pigs' left kidneys had lower weight, volume, and cortical-medullar ratio and $24.6 \%$ less glomeruli compared to the right kidney. A negative correlation was found between warm ischemia time and glomerular number.

Conclusions: About one quarter of glomeruli was lost after 30 minutes of renal warm ischemia. No glomeruli loss was detected before 20 minutes of warm ischemia. However, progressive glomerular loss was associated with increasing warm ischemia time.
\end{abstract}

Key words: Kidney. Nephrons. Nephrectomy. Warm Ischemia. Swine. 


\section{Introduction}

Partial nephrectomy provides oncological outcomes comparable to those of radical surgery of small renal cell masses as well as better preservation of renal function ${ }^{1}$. Thus, partial nephrectomy is considered the gold standard for treating localized renal tumors ${ }^{2,3}$. Warm renal ischemia is commonly performed during partial nephrectomy to achieve a bloodless surgical field, however renal ischemia has been associated with renal function impairment ${ }^{4}$.

Warm ischemia during laparoscopic partial nephrectomy is considered the most negative modifiable factor for this kind of surgery ${ }^{5-7}$. Traditionally, 30 minutes is considered the maximum safe time for renal warm ischemia. However, recent literature recommends that efforts should be made to reduce warm ischemia time to the shortest time possible time ${ }^{8}$. Some authors suggest that the time should be preferably kept below 20 or 25 minutes in order to avoid postoperative acute renal failure, because every minute counts when the renal hilum is clamped $^{8,9}$.

Most studies assessing the impact of warm ischemia on the kidney were based on functional tests such as serum creatinine level and glomerular filtration rate re-12. $^{12}$ Few researchers have used quantitative morphological methods to investigate the effects of renal warm ischemia in experimental models. They found that warm ischemia reduced the number and density of glomeruli ${ }^{13,14}$. However, they did not investigate glomerular loss relative to warm ischemia time. The hypothesis of the present study was that the longer the renal warm ischemia time, the higher the glomerular loss. Thus, the objective of this study was to investigate the number of glomeruli after different renal warm ischemia times in a pig model, using an unbiased stereological method.

\section{- Methods}

All experiments were performed according to the Brazilian law for scientific use of animals, and this project was formally approved by the local Ethics Committee for animal experimentation (CEUA 048/2011).

Thirty-two male domestic pigs (commercial crossbreed strain) with a mean weight of $25 \mathrm{~kg}$ (about three months old) were used in this study. The animals were randomly assigned into four experimental groups of eight animals. The sham group was subjected to kidney and hilar dissection, but not to renal ischemia. Three other groups (G10, $\mathrm{G} 20$, and G30) were respectively subjected to 10,20 , or 30 minutes of renal warm ischemia.

Animals were premedicated with acepromazine $(0.05 \mathrm{mg} / \mathrm{Kg}, \mathrm{IM})$, midazolan $(0.5 \mathrm{mg} / \mathrm{Kg}, \mathrm{IM})$, and cetamine $(5.0 \mathrm{mg} / \mathrm{Kg}, \mathrm{IM})$. General anesthesia was induced with propofol $(3 \mathrm{mg} / \mathrm{kg}, \mathrm{IV})$ and maintained with isoflurane ( $2 \%$ in oxygen, given by an endotracheal tube). Under general anesthesia and by using aseptic technique, the surgical procedure involved transperitoneal laparoscopic access, as previously used in pigs ${ }^{15,16}$. The left kidney was completely exposed by dissection. Renal vessels were clamped en bloc in all groups with a laparoscopic Satinsky clamp (G10, G20, and G30), except in the sham group. After the predetermined time of ischemia, the vascular clamp was removed and the normal color of the kidney was verified. In the sham group, after dissecting the renal pedicle, the animals were maintained under anesthesia for 20 minutes without renal ischemia. The right kidneys were not manipulated during the experiment and were used as controls. The animals received analgesia for 48 hours after surgery, and food and water ad libitum six hours after surgery. 
It took up to four hours after the procedure for recovery to normal ambulation. Serum creatinine levels were determined before surgery and on postoperative days 10 and 21 in order to assess renal function.

The animals were evaluated daily for 21 days after surgery, and after this period they were euthanized by anesthetic overdose (sodium thiopental $200 \mathrm{mg} / \mathrm{kg}$ IV). The kidneys were harvested, weighed, and their volumes measured by the Scherle's method ${ }^{17,18}$; then, the organs were fixed by immersion in $4 \%$ buffered formaldehyde according to our laboratory routine. Samples were collected from the cortical region of these 64 kidneys and were processed by routine histological methods for stereological analysis. Each kidney was sectioned longitudinally and from the created cut surface, samples were collected randomly, in the same fashion as in previous studies ${ }^{14,18}$. The specimens were paraffin embedded, sectioned at $5-\mu \mathrm{m}$ thickness, and stained by hematoxylin \& eosin. The cortical-medullar ratio was estimated by using the point-counting-method according the Cavalieri principle $e^{18,19}$. The absolute cortical volume (CV) was calculated by multiplying the cortical-medullar ratio by the renal volume.

From each kidney, 25 histological fields obtained from different sections of the renal cortex were photographed under 200X magnification with a digital camera (DP70, Olympus, Tokyo, Japan) coupled to a microscope (BX51, Olympus). Glomerular volumetric density
( $\mathrm{Vv}$ [glom]), was estimated by the point-counting technique with a M42 test-system ${ }^{14,20,21}$. The testsystem was digitally superimposed pver each image by using the ImageJ software (version 1.37v, NIH, Bethesda, Marylanda, USA)

The volume weighted mean glomerular volume (VWGV), was estimated by using the point-sampled intercepts method ${ }^{14,16,18,22,23}$, analyzing 50 glomeruli per kidney.

The estimation of the total number of glomeruli per kidney was calculated by multiplying the $\mathrm{CV}$ by the $\mathrm{Vv}$ [glom] and dividing the result by the VWGV.

For all stereological parameter, left kidneys were compared to right organs of each group by using the Student's unpaired t-test. In addition, linear regression was used to examine the relationship between the number of glomeruli per kidney and the warm ischemia time.

Mean creatinine serum levels were compared using one way ANOVA. For all comparisons $\mathrm{p}<0.05$ was considered significant. Data was expressed as mean \pm standard deviation. Analyses were performed using GraphPad Prism 5.0 (GraphPad Software, San Diego, USA).

\section{- Results}

All animals recovered well from surgeries. Serum creatinine levels were not different among the studied groups (Table 1).

Table 1 - Serum creatinine levels drawn before surgery and on postoperative days 10 and 21 of pigs submitted to sham surgery or to renal ischemia for 10 (G10), 20 (G20) or 30 minutes (G30).

\begin{tabular}{lcccc}
\hline & Preoperative & $\begin{array}{c}10 \text { days } \\
\text { Postoperative }\end{array}$ & $\begin{array}{c}21 \text { days } \\
\text { Postoperative }\end{array}$ & p value \\
\hline Sham & $1.52 \pm 0.38$ & $1.10 \pm 0.10$ & $1.39 \pm 0.69$ & 0.62 \\
G10 & $1.24 \pm 0.29$ & $1.19 \pm 0.87$ & $1.03 \pm 0.16$ & 0.75 \\
G20 & $1.15 \pm 0.24$ & $0.97 \pm 0.23$ & $1.10 \pm 0.66$ & 0.83 \\
G30 & $1.13 \pm 0.32$ & $1.13 \pm 0.19$ & $1.20 \pm 0.28$ & 0.85 \\
p value & 0.21 & 0.94 & 0.57 & \\
\hline
\end{tabular}

Data expressed as mean \pm S.D. 
The weight and volume of left kidney of group G30 decreased by $6.2 \%$ and $6.3 \%$, respectively, in comparison to the right kidney. In the sham, G10, and G20 groups, no difference was observed between the weight and volume of the kidneys.

The cortical-medullar ratio and absolute cortical volume was different among left and right kidneys of G30 group alone, with the left kidney having a $2.3 \%$ and $3.7 \%$ decrease in these parameters, respectively. For the other groups, no difference was noted in these parameters. Regarding $V v[$ glom] and VWGV, no difference was found among all

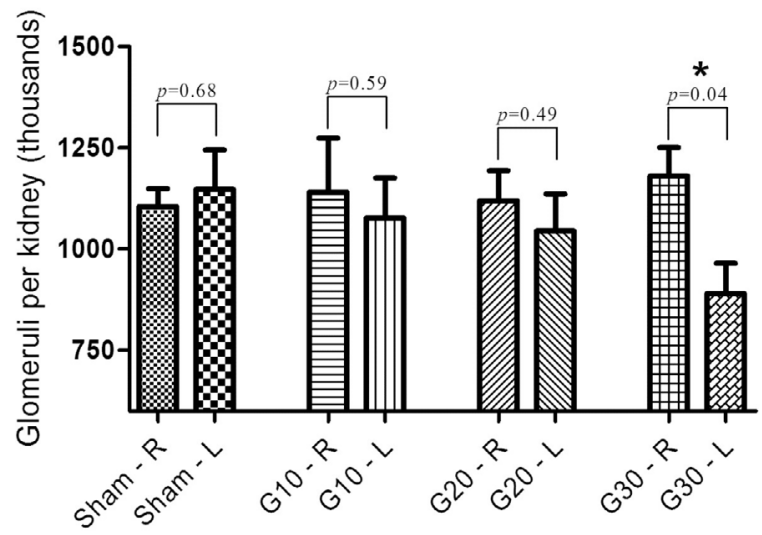

Figure 1 - Number of glomeruli in the right and left kidneys of pigs submitted to sham surgery or to left renal ischemia for 10 (G10), 20 (G20) or 30 minutes (G30). *different $(p<0.05)$ from contralateral kidney. groups.

Finally, the total number of glomeruli in left kidneys of $\mathrm{G} 30$ group decreased by $24.6 \%$ in comparison to right kidneys. This represented a loss of approximately 290.000 glomeruli caused by warm ischemia for 30 minutes (Figure 1). Although no difference was observed in the total number of glomeruli in kidneys treated with warm ischemia for 10 and 20 minutes, a negative correlation was found between the warm ischemia time and the number of glomeruli (Figure 2). All stereological data is presented in Table 2 and histological examples of all groups are presented in Figure 3.

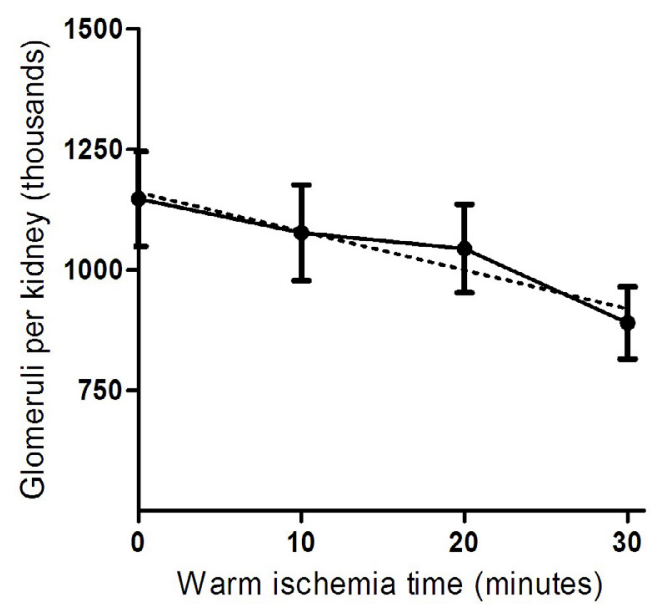

Figure 2 - Graphic showing the number of glomeruli in kidneys submitted to sham surgery or warm ischemia for 10, 20 or 30 minutes (solid line); and linear regression line (dashed line) showing the negative correlation between warm ischemia time and number of glomeruli $\left(r^{2}=0.9147\right)$.

Table 2 - Stereological data of right and left kidneys of pigs submitted to sham surgery or to left renal ischemia for 10 (G10), 20 (G20) or 30 minutes (G30).

\begin{tabular}{|c|c|c|c|c|c|c|c|c|c|c|c|c|}
\hline & \multicolumn{3}{|c|}{ Sham } & \multicolumn{3}{|c|}{ G10 } & \multicolumn{3}{|c|}{ G20 } & \multicolumn{3}{|c|}{ G30 } \\
\hline & Right & Left & p value & Right & Left & $\mathrm{p}$ value & Right & Left & $p$ value & Right & Left & p value \\
\hline Kidney weight (g) & $56.8 \pm 4.9$ & $58.2 \pm 8.5$ & 0.52 & $58.0 \pm 7.1$ & $55.9 \pm 7.5$ & 0.47 & $64.5 \pm 11.1$ & $64.0 \pm 11.5$ & 0.51 & $59.2 \pm 10.9$ & $55.5 \pm 11.0^{*}$ & 0.008 \\
\hline Kidney volume (ml) & $54.4 \pm 4.1$ & $55.2 \pm 7.7$ & 0.70 & $55.4 \pm 6.4$ & $53.1 \pm 6.5$ & 0.37 & $56.7 \pm 7.3$ & $56.8 \pm 8.2$ & 0.93 & $56.6 \pm 9.8$ & $53.0 \pm 10.5 *$ & 0.007 \\
\hline Cortical-medullar ratio (\%) & $71.6 \pm 2.3$ & $70.4 \pm 4.1$ & 0.32 & $71.8 \pm 1.8$ & $72.1 \pm 2.1$ & 0.48 & $72.3 \pm 5.5$ & $71.6 \pm 3.9$ & 0.39 & $71.8 \pm 2.4$ & $70.1 \pm 2.2^{*}$ & 0.04 \\
\hline Cortical volume (ml) & $38.9 \pm 3.3$ & $38.9 \pm 6.5$ & 0.98 & $39.8 \pm 4.8$ & $38.3 \pm 4.6$ & 0.38 & $41.0 \pm 5.9$ & $40.7 \pm 6.5$ & 0.82 & $40.6 \pm 7.4$ & $37.2 \pm 7.9^{*}$ & 0.002 \\
\hline Vv[glom] (\%) & $3.79 \pm 0.5$ & $3.72 \pm 0.5$ & 0.68 & $3.95 \pm 1.0$ & $3.82 \pm 0.7$ & 0.61 & $3.83 \pm 0.6$ & $3.57 \pm 0.6$ & 0.30 & $3.59 \pm 0.4$ & $3.08 \pm 0.9$ & 0.16 \\
\hline 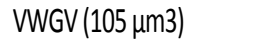 & $13.4 \pm 1.6$ & $12.7 \pm 1.1$ & 0.25 & $14.1 \pm 2.6$ & $13.9 \pm 2.5$ & 0.76 & $14.1 \pm 2.4$ & $14.1 \pm 2.1$ & 0.97 & $12.5 \pm 2.4$ & $12.4 \pm 1.2$ & 0.94 \\
\hline Glomeruli (millions) & $1.10 \pm 0.1$ & $1.14 \pm 0.2$ & 0.68 & $1.14 \pm 0.3$ & $1.07 \pm 0.3$ & 0.59 & $1.12 \pm 0.2$ & $1.04 \pm 0.2$ & 0.49 & $1.18 \pm 0.2$ & $0.89 \pm 0.2^{*}$ & 0.04 \\
\hline
\end{tabular}

Data expressed as mean \pm S.D. * different $(p<0.05)$ from contralateral kidney. 


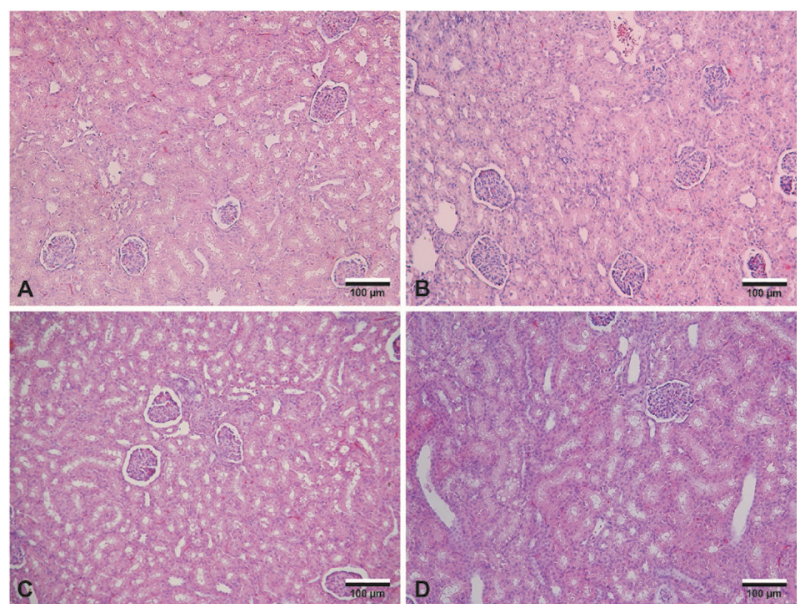

Figure 3 - Photomicrographs from renal cortex of pigs submitted to sham surgery (A), 10 minutes (B), 20 minutes (C), and 30 minutes of warm ischemia (D). Hematoxylin and eosin, $x 200$.

\section{- Discussion}

Warm ischemia was recently mentioned as the ultimate enemy for partial nephrectomy 6 . There are conflicting opinions in the literature on the suggested maximum safe time for renal warm ischemia. The optimal time has been stated as 20, 25 or 30 minutes or even longer than 30 minutes by some authors ${ }^{24,25}$. Although the maximum time for a safe warm ischemia is controversial, the consensus is that it is an important aspect during partial nephrectomy. The results of the present experiment showed that a 30-minute period of renal warm ischemia led to a significant decrease in the number of glomeruli. Therefore, the use of warm ischemia for 30 minutes or more is discouraged, and this is in accordance with the authors who recommend the time for safe warm ischemia to be lower than 30 minutes ${ }^{24,25}$.

Animals subjected to 10 or 20 minutes of warm ischemia did not show a statistically significant alteration of the glomerular number. This suggests that 20 minutes of warm ischemia could be considered as a "statistically safe" period for renal warm ischemia in the pig model. However, one may note that the kidneys subjected to 20 minutes of warm ischemia had 75.000 less glomeruli than the contralateral organs not subjected to ischemia. In addition, kidneys exposed to only 10 minutes of warm ischemia lost 64.000 glomeruli. Although the glomerular loss after 10 and 20 minutes of renal warm ischemia was not statistically significant, linear regression demonstrated a progressive decrease in functional nephrons the longer the kidney remains under warm ischemia. It supports the statement that "every minute counts/matters" when the renal hilum is clamped during partial nephrectomy ${ }^{8,25}$.

However, there is no published information about how much a minute counts for a kidney under warm ischemia. We have used stereology to quantify the number of glomeruli in the kidney, and correlated this number to warm ischemia time. Linear regression indicated that 8.000 glomeruli were lost per minute during warm ischemia.

Stereological quantification was used to precisely quantify the number of glomeruli. Stereological methods have been used to determine the glomerular number in kidneys affected by pneumoperitoneum, diabetes, and chronic stress ${ }^{19,22,26}$. Stereological glomeruli quantification has also been used to study renal damage after partial nephrectomy ${ }^{14}$ or for comparing arteriovenous with arterial renal ischemia ${ }^{13}$. As the goal of partial nephrectomy is to remove renal tumors sparing as much nephrons as possible, it is reasonable that an unbiased determination of the glomerular number is important for studying the impact on the kidney. To our knowledge, this study is the first of its kind to show that warm ischemia time reduces the number of glomeruli and consequently the number of nephrons in the kidney.

The serum creatinine levels remained unaffected even though there was a significant reduction in glomeruli in kidneys subjected to 30 minutes of warm ischemia. This suggests that using serum creatinine levels as single method for studying the impact of ischemia is 
flawed. Quantitative morphological methods are preferable. However, as indicated by another study, the serum creatinine levels may be normal because the right kidney was unaffected $^{27}$. Hence, a single kidney model should be used for studies measuring global renal function after warm ischemia ${ }^{28}$.

Several methods have been proposed for increasing the ischemia time without affecting the kidney, some of which are commonly used in clinical practice. These include arterial or selective clamping instead of arteriovenous clamping, use of renal protective drugs, and cold renal ischemia. However, most of these methods are recommended based on functional tests only, with little quantitative morphological evidence.

A limitation of this study is that the kidney damage due to warm ischemia cannot be directly transposed to the damage that may occur in humans. Although the pig model is the most similar to human anatomy and physiology ${ }^{15,29}$, this is still an experimental setting and different from the clinical setting. These animals were healthy, without renal tumors or any other medical condition. The pigs were not subjected to partial nephrectomy, but only to warm ischemia. However, we can imagine that in a clinical scenario the glomerular loss would be even higher after partial nephrectomy than what was observed in this study.

\section{Conclusions}

About one quarter of the glomeruli is lost after 30 minutes of renal warm ischemia in a pig model. No significant glomerular loss occurs at 10 or 20 minutes of warm ischemia. There is a progressive loss of kidney glomeruli relative to warm ischemia time. This confirms that every minute counts when dealing with a kidney under warm ischemia.

\section{References}

1. Mashni JW, Assel M, Maschino A, Russo M, Masi B, Bernstein M, Huang WC, Russo P. New chronic kidney disease and overall survival after nephrectomy for small renal cortical tumors. Urology. 2015 Dec;86(6):1137-43. doi: 10.1016/j.urology.2015.07.046.

2. Campbell SC, Novick AC, Belldegrun A, Blute ML, Chow GK, Derweesh IH, Faraday MM, Kaouk JH, Leveillee RJ, Matin SF, Russo P, Uzzo RG. Guideline for management of the clinical T1 renal mass. J Urol. 2009 Oct;182(4):1271-9. doi: 10.1016/j. juro.2009.07.004.

3. Ljungberg $B$, Bensalah $K$, Canfield $S$, Dabestani S, Hofmann F, Hora M, Kuczyk MA, Lam T, Marconi L, Merseburger AS, Mulders P, Powles T, Staehler M, Volpe A, Bex A. EAU guidelines on renal cell carcinoma: 2014 update. Eur Urol. 2015 May;67(5):913-24. doi: 10.1016/j.eururo.2015.01.005.

4. George AK, Herati AS, Srinivasan AK, Rais-Bahrami S, Waingankar N, Sadek MA, Schwartz MJ, Okhunov Z, Richstone L, Okeke Z, Kavoussi LR. Perioperative outcomes of off-clamp vs complete hilar control laparoscopic partial nephrectomy. BJU Int. 2013 Apr;111(4 Pt B):E235-41. doi: 10.1111/j.1464-410X.2012.11573.x.

5. Lane BR, Russo P, Uzzo RG, Hernandez AV, Boorjian SA, Thompson RH, Fergany AF, Love TE, Campbell SC. Comparison of cold and warm ischemia during partial nephrectomy in 660 solitary kidneys reveals predominant role of nonmodifiable factors in determining ultimate renal function. J Urol. 2011 Feb;185(2):421-7. doi: 10.1016/j. juro.2010.09.131.

6. Pignot G, Bouliere F, Patard JJ. Warm ischaemia: the ultimate enemy for partial nephrectomy? Eur Urol. 2010Sep;58(3):3379. doi: 10.1016/j.eururo.2010.07.002.

7. Zhang Z, Zhao J, Dong W, Remer E, Li J, Demirjian S, Zabell J, Campbell SC. Acute kidney injury after partial nephrectomy: role of parenchymal mass reduction and ischemia and impact on subsequent functional recovery. Eur Urol. 2016 Apr;69(4):745-52. doi: 10.1016/j.eururo.2015.10.023.

8. Thompson $\mathrm{RH}$, Lane BR, Lohse $\mathrm{CM}$, Leibovich BC, Fergany A, Frank I, Gill IS, Blute ML, Campbell SC. Every minute counts when the renal hilum is clamped during partial 
nephrectomy. Eur Urol. 2010 Sep;58(3):3405. doi: 10.1016/j.eururo.2010.05.047.

9. Becker F, Van Poppel H, Hakenberg OW, Stief C, Gill I, Guazzoni G, Montorsi F, Russo P, Stockle M. Assessing the impact of ischaemia time during partial nephrectomy. Eur Urol. 2009 Oct;56(4):625-34. doi: 10.1016/j.eururo.2009.07.016.

10.Lane BR, Fergany AF, Weight CJ, Campbell SC. Renal functional outcomes after partial nephrectomy with extended ischemic intervals are better than after radical nephrectomy. J Urol. 2010 Oct;184(4):128690. doi: 10.1016/j.juro.2010.06.011.

11.Ramirez D, Caputo P, Krishnan J, Zargar H, Kaouk JH. Robotic partial nephrectomy with intracorporeal renal hypothermia using ice slush: step-by-step technique and matched comparison to warm ischemia. BJU Int. 2016 Mar;117(3):531-6. doi: 10.1111/bju.13346.

12.Zhang Z, Zhao J, Velet L, Ercole CE, RemerEM, Mir CM, Li J, Takagi T, Demirjian S, Campbell SC. Functional recovery from extended warm ischemia associated with partial nephrectomy. Urology. 2016 Jan;87:106-13. doi: 10.1016/j.urology.2015.08.034.

13.Bagetti-Filho HJ, Sampaio FJ, Marques RG, Pereira-Sampaio MA. Different from renal artery only clamping, artery and vein clamping causes a significant reduction in number of rat glomeruli during warm ischemia. J Endourol. 2012 Oct;26(10):13359. doi: 10.1089/end.2012.0166.

14.de Souza DB, de Oliveira LL, da Cruz MC, Abilio EJ, Costa WS, Pereira-Sampaio MA, Sampaio FJ. Laparoscopic partial nephrectomy under warm ischemia reduces the glomerular density in a pig model. J Endourol. 2012 Jun;26(6):706-10. doi: 10.1089/end.2011.0412.

15.de Souza DB, Abilio EJ, Costa WS, Sampaio MA, Sampaio FJ. Kidney healing after laparoscopic partial nephrectomy without collecting system closure in pigs. Urology. 2011 Feb;77(2):508 e5-9. doi: 10.1016/j. urology.2010.08.017.

16.Bechara GR, Damasceno-Ferreira JA, Abreu LA, Costa WS, Sampaio FJ, PereiraSampaio MA, Souza DB. Glomerular loss after arteriovenous and arterial clamping for renal warm ischemia in a swine model. Acta Cir Bras. 2016 Nov;31(11):753-8. doi: 10.1590/S0102-865020160110000008.

17. Ribeiro CT, Milhomem R, De Souza DB, Costa WS, Sampaio FJ, Pereira-Sampaio MA. Effect of antioxidants on outcome of testicular torsion in rats of different ages. J Urol. 2014 May;191(5 Suppl):1578-84. doi: 10.1016/j. juro.2013.09.066.

18.Abreu LA, Damasceno-Ferreira JA, Costa WS, Pereira-Sampaio M, Sampaio FJ, de souza D. Glomerular loss after renal radiofrequency ablation are comparable to 30 minutes of warm ischemia. J Endourol. $2017 \mathrm{Apr} 18$. doi: 10.1089/end.2016.0899. [Epub ahead of print]

19.Souza DB, Costa WS, Cardoso LE, Benchimol M, Pereira-Sampaio MA, Sampaio FJ. Does prolonged pneumoperitoneum affect the kidney? Oxidative stress, stereological and electron microscopy study in a rat model. Int Braz J Urol. 2013 Jan-Feb;39(1):30-6. doi: 10.1590/S1677-5538.

20.Bechara GR, de Souza DB, Simoes $M$, Felix-Patricio B, Medeiros JL, Jr., Costa WS, Sampaio FJ. Testicular morphology and spermatozoid parameters in spontaneously hypertensive rats treated with enalapril. J Urol. 2015 Nov;194(5):1498-503. doi: 10.1016/j.juro.2015.06.073.

21.de Souza DB, Silva D, Cortez CM, Costa WS, Sampaio FJ. Effects of chronic stress on penile corpus cavernosum of rats. J Androl. 2012 Jul-Aug;33(4):735-9. doi: 10.2164/ jandrol.111.014225.

22. Benchimol deSouza D, Silva D, Marinho Costa Silva C, Barcellos Sampaio FJ, Silva Costa W, Martins Cortez C. Effects of immobilization stress on kidneys of Wistar male rats: a morphometrical and stereological analysis. Kidney Blood Press Res. 2011 34(6):424-9. doi: 10.1159/000328331.

23.Bechara G, Damasceno-Ferreira JA, Abreu LA, Costa WS, Sampaio FJ, Pereira-Sampaio $M$, de souza $D$. Glomerular morphology and renal function after warm ischemia by main artery or selective clamping in a porcine model. Urol Int. 2017 Mar 30. doi: 
10.1159/000458762. [Epub ahead of print]

24. Kim SP, Thompson RH. Kidney function after partial nephrectomy: current thinking. Curr Opin Urol. 2013 Mar;23(2):105-11. doi: 10.1097/MOU.0b013e32835d8ec1.

25.Patel AR, Eggener SE. Warm ischemia less than 30 minutes is not necessarily safe during partial nephrectomy: every minute matters. Urol Oncol. 2011 Nov-Dec;29(6):826-8. doi: 10.1016/j.urolonc.2011.02.015.

26. Hokke SN, Armitage JA, Puelles VG, Short KM, Jones L, Smyth IM, Bertram JF, CullenMcEwen LA. Altered ureteric branching morphogenesis and nephron endowment in offspring of diabetic and insulin-treated pregnancy. PloS One. 2013;8(3):e58243. doi: 10.1371/journal.pone.0058243.

27. Berczi C, Thomas B, Bacso Z, Flasko T. Long- term oncological and functional outcomes of partial nephrectomy in solitary kidneys. Clin Genitourin Cancer. 2016 Jun;14(3):e275-81. doi: 10.1016/j.clgc.2015.11.014.

28.Thompson $\mathrm{RH}$, Lane $\mathrm{BR}$, Lohse $\mathrm{CM}$, Leibovich BC, Fergany A, Frank I, Gill IS, Campbell SC, Blute ML. Comparison of warm ischemia versus no ischemia during partial nephrectomy on a solitary kidney. Eur Urol. 2010 Sep;58(3):331-6. doi: 10.1016/j. eururo.2010.05.048.

29.Pereira-Sampaio MA, Favorito LA, Sampaio FJ. Pig kidney: anatomical relationships between the intrarenal arteries and the kidney collecting system. Applied study for urological research and surgical training. J Urol. 2004 Nov;172(5 Pt 1):2077-81. PMID: 15540793.

\section{Correspondence:}

Diogo Benchimol de Souza

Unidade de Pesquisa Urogenital-UERJ

Avenida 28 de Setembro, 87, Fundos

20551-030 Rio de Janeiro - RJ Brasil

Tel.: (55 21)2868-8399

diogobenchimol@gmail.com

Received: Jan 05, 2017

Review: Mar 06, 2017

Accepted: Apr 07, 2017
Conflict of interest: none

Financial sources: CNPq, CAPES, FAPERJ
${ }^{1}$ Research performed at Experimental Farm, Faculty of Veterinary Medicine, Universidade Federal Fluminense (UFF), Cachoeiras de Macacu-RJ, and Urogenital Research Unit, Universidade Estadual do Rio de Janeiro (UERJ), Brazil. 